\title{
Hybrid top-down/bottom-up fabrication of regular arrays of AIN nanorods for deep-UV core-shell LEDs
}

\author{
P.-M. Coulon ${ }^{*}, 1$, G. Kusch², E.D. Le Boulbar ${ }^{1}$, P. Chausse ${ }^{1}$, C. Bryce ${ }^{2}$, R.W. Martin ${ }^{2}$ and P.A. Shields ${ }^{1}$ \\ ${ }^{1}$ Centre of Nanoscience \& Nanotechnology, University of Bath, BA2 7AY, UK \\ Department of Electronic and Electrical Engineering, University of Bath, BA2 7AY, UK \\ 2 Department of Physics, SUPA, University of Strathclyde, G4 0NG, UK
}

Received ZZZ, revised ZZZ, accepted ZZZ

Published online ZZZ (Dates will be provided by the publisher.)

Keywords AlN, nanorod, top-down etching, MOVPE

* Corresponding author: e-mail P.Coulon@bath.ac.uk

Core-shell nanostructures are predicted to highly improve the efficiency of deep-UV LEDs, owing to their low defect density, reduced quantum-confined Stark effect, highquality non-polar growth and improved extraction efficiency. In this paper, we report on the nanofabrication of high-quality AIN nanorod arrays using a hybrid topdown/bottom-up approach for use as a scaffold for UV LED structures. We describe the use of Displacement Talbot Lithography to fabricate a metallic hard etch mask to allow AlN nanorod arrays to be dry etched from a planar AlN template. In particular, we investigate the impact of etching parameters on the nanorod etch rate, tapering profile and mask selectivity in order to achieve vertical-sided nanorod arrays with high aspect ratios. AlN facet recovery is subsequently explored by means of regrowth using Metal Organic Vapor Phase Epitaxy. Low pressure and high V/III ratio promote straight and smooth sidewall faceting, which results in an improvement of the optical quality compared to the initial AIN template. The promising results open new perspectives for the fabrication of highefficiency deep-UV-emitting core-shell LEDs.
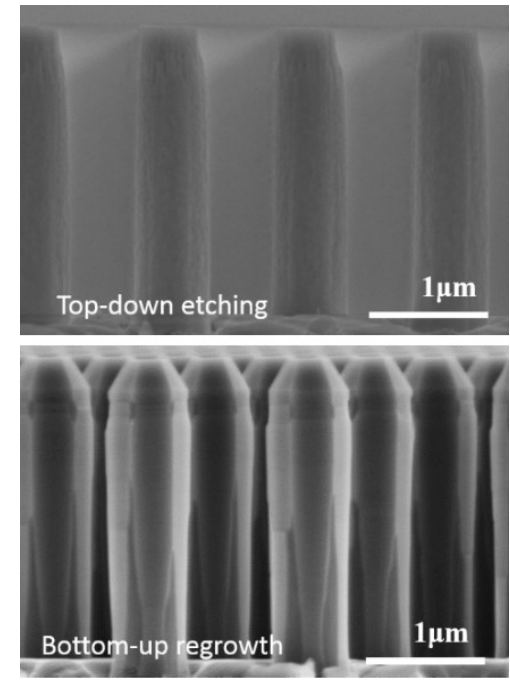

AlN nanorod arrays after top-down etching and bottom-up regrowth
1 Introduction Compared to current ultra-violet (UV) technology, mostly based on mercury lamps, III-nitride UVbased LEDs have the advantage of being compact, robust, and environmentally friendly with the potential to exhibit a long life time as well as requiring low voltage and electrical power. As such, III-nitride UV-based LEDs are considered a reliable alternative for a broad range of UV applications including water purification, surface decontamination, optical sensing, and solid-state lighting [1]. Whereas much effort around the world has been devoted to improve the external quantum efficiency (EQE) of InGaN-based blue LEDs, with an EQE now reaching $84 \%$ in laboratories [2] and $69 \%$ for commercialized blue LEDs, $\mathrm{Al}_{\mathrm{x}} \mathrm{Ga}_{\mathrm{x}-1} \mathrm{~N}$ UV based LEDs barely reach a maximum of $30 \% \mathrm{EQE}$ in the
UVA spectral region, falling to $1-3 \%$ in the UVB and UVC [1]. Several factors contribute to these low values such as the high defect densities in AlN and AlGaN materials [3], the low carrier injection efficiency and the poor light extraction.

The use of 3D nanostructures has the potential to circumvent the key problems existing in UV planar 2D technology. However, a key obstacle to their implementation in UV devices is the immaturity of techniques to grow such structures in $\mathrm{Al}_{\mathrm{x}} \mathrm{Ga}_{\mathrm{x}-1} \mathrm{~N}$-based materials. For example, whereas the selective area growth (SAG) of GaN nanorod arrays has been demonstrated by metal organic vapor phase epitaxy (MOVPE) on various substrates $[4,5,6]$, the SAG of AlN and Al-rich AlGaN nanorods has not been achieved 
yet due to the low diffusion length of Al-adatoms [7]. Instead, the use of a combined top-down etching and MOVPE regrowth approach, already demonstrated for $\mathrm{GaN} / \mathrm{InGaN}$ core-shell nanorod arrays [8], represents a promising route towards the fabrication of AlN/AlGaN core-shell devices [9]. This approach will allow 1) the reduction of the defect density compared to the initial template by means of dislocation filtering [10], 2) an increase of the overall area of emission via the regrowth of the active region shell on the nanorod core [11],3) the avoidance of the strong spontaneous and piezoelectric polarization fields through regrowth on high quality $m$-plane non-polar facets, and 4) improved light extraction efficiency through the nanorod array geometry [12]. However, prior to creating device structures with active quantum wells, the fabrication of AIN nanorod arrays with vertical sidewalls and well-defined facets should be demonstrated.

This paper presents a detailed study on the top-down etching of AlN nanorod arrays and the subsequent bottomup MOVPE regrowth. The etch rate and tapering (i.e. the degree of verticality of the sidewall facets) is found to be different from $\mathrm{GaN}$ nanorods and highly dependent on the etching conditions. The MOVPE facet recovery is improved at low pressure and high V/III ratio or with the use of a TMAl pulsed growth mode. It is suggested that an improvement in the aspect ratio and surface roughness after etching can lead to a complete and successful facet recovery. This represents the first step towards achieving deep-UV-emitting core-shell LEDs.

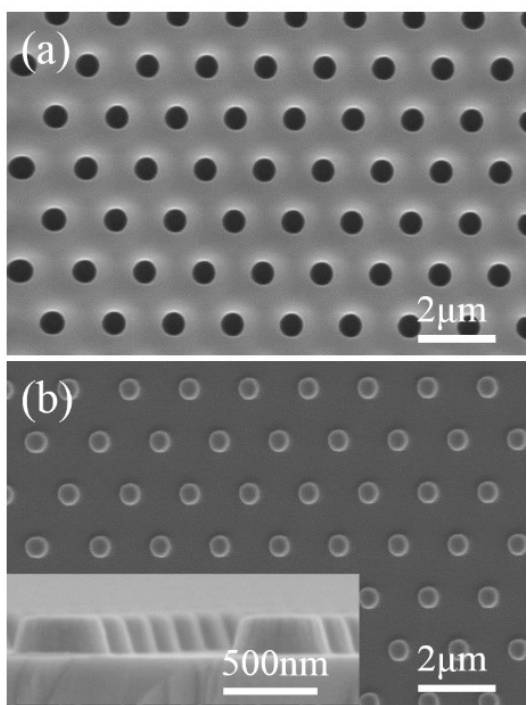

Figure 1 (a) Secondary-electron SEM images of the hole pattern in resist after DTL exposure and development. (b) Plan view and cross section (inset) images of the $\mathrm{Au} / \mathrm{Ni}$ metal dot mask after liftoff.

\section{Experimental methods}

2.1 Metal mask fabrication The AlN templates grown by MOVPE on (0001) sapphire substrates were sourced commercially (Nanowin) and consisted of a thickness of $\sim 4-5$ $\mu \mathrm{m}$ [13]. The fabrication of a metal dot mask was achieved by a lift-off process using Displacement Talbot lithography (DTL) (PhableR 100, Eulitha). A bottom antireflective layer (BARC) (AZ BARLI II) was spun on the templates to obtain a layer thickness of $290 \mathrm{~nm}$, followed by a $400 \mathrm{~nm}$ layer of high-contrast positive resist (Ultra- $i$ 123, Dow). The resist was subsequently exposed with a dose of $160 \mathrm{~mJ} . \mathrm{cm}^{-2}$ through an amplitude mask comprising holes with a diameter of about $800 \mathrm{~nm}$ in a hexagonal pattern with a $1.5 \mu \mathrm{m}$ pitch. Figure 1.a shows the pattern obtained in the resist after DTL exposure and development, which consisted of an array of $\sim 600 \mathrm{~nm}$ diameter openings. Finally, $10 \mathrm{~nm} \mathrm{Au}$ and $200 \mathrm{~nm}$ Ni layers were deposited via e-beam evaporation and subsequent lift-off was achieved with MF-CD-26 developer. The gold layer is used to prevent the formation of Ga$\mathrm{Ni}$ compound on the top c-plane, which could lead to the incorporation of undesired impurity in the MOVPE growth reactor. This fabrication process allowed the creation of a homogeneous $1.5 \mu \mathrm{m}$ hexagonal array of $\mathrm{Au} / \mathrm{Ni}$ metal dots of diameter $~ 550-600 \mathrm{~nm}$, as shown in Figure 1.b on a full $50 \mathrm{~mm}$ wafer.

Table 1 Plasma properties, etch rate and selectivity of the etching experiments.

\begin{tabular}{|c|c|c|c|c|c|c|}
\hline $\begin{array}{l}\text { Exp. } \\
\text { No. }\end{array}$ & $\begin{array}{l}\text { Etch time } \\
(\mathrm{min})\end{array}$ & $\begin{array}{l}\text { Pressure } \\
(\mathrm{Pa})\end{array}$ & $\begin{array}{l}\text { RF } \\
(\mathrm{W})\end{array}$ & $\begin{array}{l}\text { DC Bias } \\
(\mathrm{V})\end{array}$ & $\begin{array}{l}\text { Etch } \\
(\mathrm{nm} /\end{array}$ & Sele \\
\hline 1 & 14 & 1.2 & 80 & 328 & 185 & $26: 1 \pm 10 \%$ \\
\hline 2 & 14 & 1.6 & 80 & 349 & 125 & $21 \%$ \\
\hline 3 & 14 & 2 & 80 & 366 & 97 & $90: 1 \pm 66 \%$ \\
\hline 4 & 14 & 2 & 100 & 408 & 110 & $50: 1 \pm 33 \%$ \\
\hline 5 & 14 & 2 & 120 & 447 & 123 & $28: 1 \pm 17 \%$ \\
\hline 6 & 14 & 1.8 & 100 & 403 & 125 & $44: 1 \pm 26 \%$ \\
\hline 7 & 21 & 1.8 & 100 & 403 & 123 & $31: 1 \pm 12 \%$ \\
\hline 8 & 30 & 1.8 & 100 & 404 & 133 & $20: 1 \pm 5 \%$ \\
\hline
\end{tabular}

2.2 Top-down ICP etching The impact of the dry etching conditions were studied in an inductively coupled plasma (ICP) dry etch system (Oxford Instruments System 100 Cobra). The metal-dot-patterned samples were cleaned in a 2 min reactive-ion etching (RIE) oxygen plasma to remove any BARC residue. Prior to nanorod etching, a $30 \mathrm{sec}$ $\mathrm{BCl}_{3} / \mathrm{Ar}$ pretreatment step was performed to remove the aluminium oxide $\left(\mathrm{Al}_{2} \mathrm{O}_{3}\right)$ formed at the surface of the AlN layers [14]. The variation of one plasma etching parameter was studied while keeping all other conditions fixed, as detailed in Table 1. The experiments were performed for a fixed $\mathrm{Cl}_{2} / \mathrm{Ar}$ chemistry of $50 \mathrm{sccm} / 10 \mathrm{sccm}$, a temperature set to $150^{\circ} \mathrm{C}$ and an ICP source power of $800 \mathrm{~W}$. Argon contributes to efficient decomposition and ionization of the chlorine feed gas and plays the primary role in removing the etch by-products [15]. Our standard GaN etching recipe involves the use of high temperature etching [16], which has been demonstrated to greatly enhance the verticality of nanorods. 
2.3 Bottom-up MOVPE regrowth The bottom-up regrowth was carried out in a 1 x 2 " horizontal Aixtron MOVPE reactor using various growth conditions. To study the influence of several growth parameters on the facet recovery during regrowth, different sample sets were grown, so that within each set only one growth parameter was changed while other parameters were fixed, as detailed in Table 2. Different pulsed growth modes were also explored such as $\mathrm{NH}_{3}$ pulsed epitaxy, modified migration-enhanced epitaxy (MEE) where the fluxes are supplied in turn with a simultaneous supply inserted in between the MEE [7], and TMAl pulsed epitaxy. The characteristics of each growth mode are presented in Figure 2. $\mathrm{H}_{2}$ was used as the carrier gas and fixed at $8 \mathrm{slm}$. The values stated for the $\mathrm{V} / \mathrm{III}$ ratios in Table 2 for the pulsed modes correspond to times when both precursors were injected into the growth reactor.

Table 2 Growth parameters employed for the different samples.

\begin{tabular}{lllll}
\hline $\begin{array}{l}\text { Exp. } \\
\text { No. }\end{array}$ & Growth mode & $\begin{array}{l}\text { Temperature } \\
\left({ }^{\circ} \mathrm{C}\right)\end{array}$ & $\begin{array}{l}\text { Pressure } \\
(\mathrm{mbar})\end{array}$ & $\mathrm{V} / \mathrm{III}$ ratio \\
\hline 1 & Continuous & 1125 & 50 & 74.4 \\
2 & Continuous & 1025 & 50 & 74.4 \\
3 & Continuous & 1125 & 50 & 3819 \\
4 & Continuous & 1125 & 20 & 3819 \\
5 & Continuous & 1125 & 20 & 15277 \\
6 & Continuous & 1125 & 20 & 30554 \\
7 & $\mathrm{NH}_{3}$ pulsed & 1125 & 20 & 30554 \\
8 & $\mathrm{MEE}$ & 1125 & 20 & 30554 \\
9 & $\mathrm{TMAl}$ pulsed & 1125 & 20 & 30554 \\
\hline
\end{tabular}

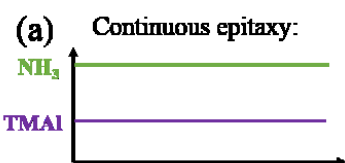

(b) $\mathrm{NH}_{3}$ pulsed epitaxy:

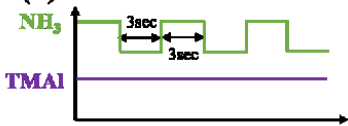

(c) Modified MEE:

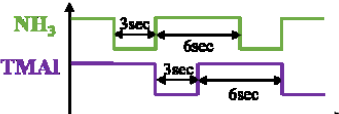

(d) TMAl pulsed epitaxy:

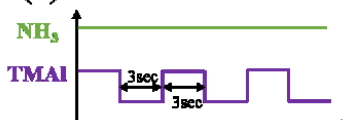

Figure 2 Schematics of the different growth-mode strategies: (a) Continuous epitaxy, (b) $\mathrm{NH}_{3}$ pulsed epitaxy, (c) Modified migration-enhanced epitaxy (MEE), (d) TMAl pulsed epitaxy.

2.4 Characterization The characteristics of the nanorod array as a function of the plasma properties and MOVPE regrowth conditions were monitored by scanning electron microscopy (SEM). Cross-sectional images of nanorods were used to extract the nanorod characteristics after the etching experiments (tapering profile, etch rate and the selectivity) and MOVPE regrowth (growth rate and dimensions).

The optical emission properties of the AlN nanorods were assessed by room temperature high resolution cathodoluminescence (CL) hyperspectral imaging. Light was collected using an NA0.28 reflecting objective with its axis perpendicular to the electron beam and focused directly to the entrance of the spectrograph using an off-axis paraboloidal mirror. Acquisition were performed at $15 \mathrm{kV}$, $1 \mathrm{mbar}$ with a $125 \mathrm{~mm}$ focal length spectrograph having a 600 lines/mm grating and a $50 \mu \mathrm{m}$ entrance slit, coupled to a cooled electron-multiplying charge-coupled device (EMCCD) detector [17].

\section{Results and discussion}

3.1 Impact of etching conditions: pressure Figure 3 shows SEM images of AlN nanorods etched for various pressures. The increase of pressure induces a decrease in the etch rate from $185 \mathrm{~nm} / \mathrm{min}$ to $97 \mathrm{~nm} / \mathrm{min}$ (Table 1), a better selectivity (Table 1) and a change in the nanorod tapering profile from inclined to straight sidewalls. Note, the selectivity is the ratio between the AlN etch rate and $\mathrm{Ni}$ etch rate. In all experiments, the $\mathrm{Ni}$ etch rate was extracted from the middle of the Ni dot thus does not take into account the rounding of the $\mathrm{Ni}$ dot at the dot edges.
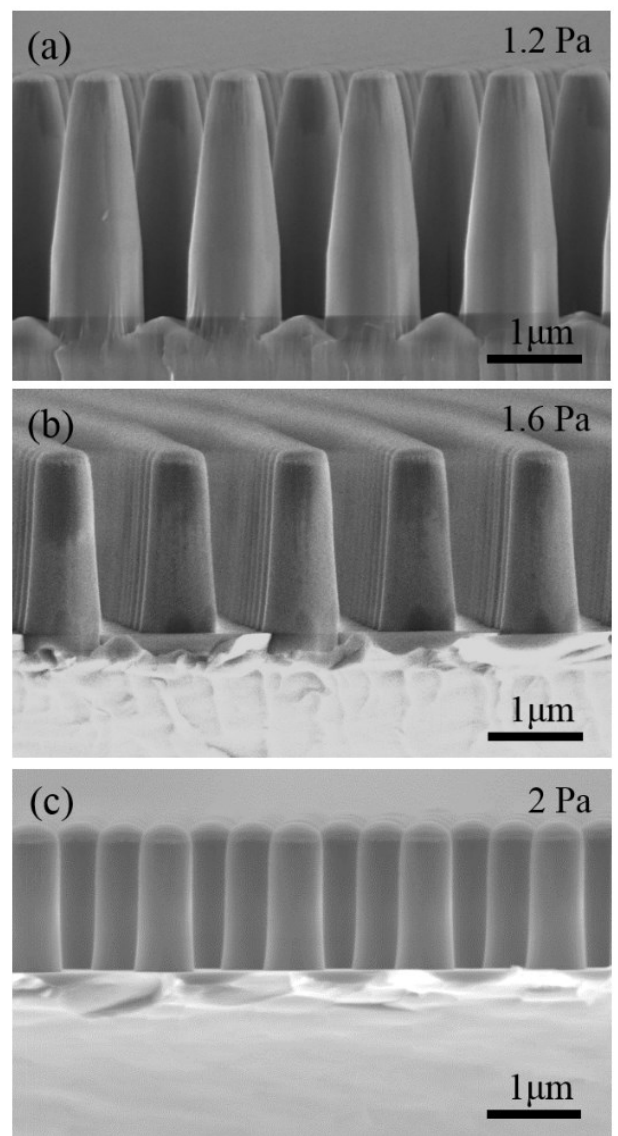

Figure 3 Cross-section SEM images of AIN nanorod arrays etched at different pressure.

The pressure predominantly affects the etch rate through the change in the incident ion energy via the mean free path and the DC bias on the sample electrode. Since the chemical etching component is assisted by the surface bombardment by ions of the AlN, a reduced ion energy at higher pressures reduces the overall etch rate as a result of the reduced mean 
free path, despite the small increase in DC bias. However, a lower ion energy also leads to a reduced physical sputtering component of the etch, leading to an overall increased selectivity. Aditionally, the pressure affects the sidewall profile through its influence on the range of incident angles of the ions. A greater range at the higher pressures caused by gas-phase collisions aids the sputtering of etch byproducts on the nanorod sidewalls that passivate the surface and restrict the lateral etch rate.

Therefore, the increase of the pressure is predicted to reduce the tapering profile through lower erosion of the hard mask and improved chemical etching of the sidewalls.

3.2 Impact of etching conditions: RF power Figure 4 displays the effect of RF power on the etched AlN nanorods. It can be observed that with the increase in RF power the AlN etch rate increases from $97 \mathrm{~nm} / \mathrm{min}$ to 123 $\mathrm{nm} / \mathrm{min}$ (Table 1), the selectivity decreases (Table 1) and the tapering profile of the nanorod barely changes. Indeed, only a slightly more pronounced undercut (i.e a reduction of the nanorod diameter around mid-height) of the nanorod can be observed at higher RF power.
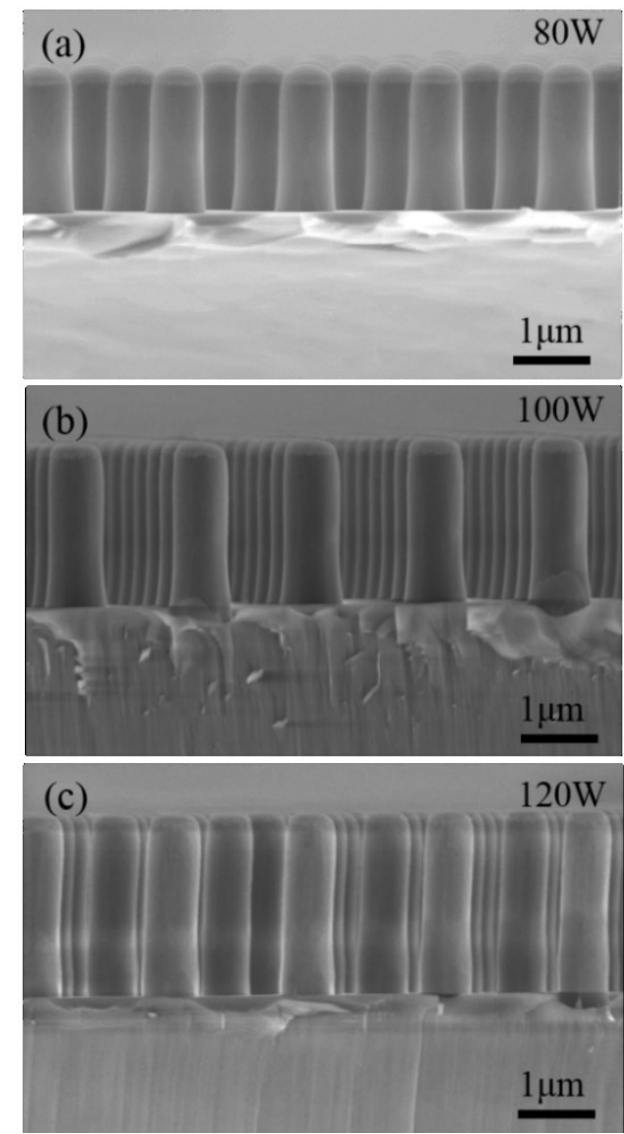

Figure 4 Cross-section SEM images of AlN nanorod arrays etched at different RF powers.

As presented in Table 1, an increase of the RF power leads to a significantly higher DC bias. Since the DC bias controls the ion energy, this leads to the higher RF power enhancing the physical sputtering component. This aids the removal of etch byproducts, assists in bond breaking and enhances chemical reactions at the surface which increases the formation of volatile etch products and thus improves the chemical etching component. Thus, an increase of the RF power enhances both the physical as well as the chemical component of etching, leading to a higher etch rate.

Similar etching conditions as those used to achieve the AlN nanorod etching experiment 5 (Figure 4.c) were employed in our previous work to etch GaN nanorods [16]. When comparing the etch characteristics between AIN and GaN nanorods, a decrease in the etch rate and an increase in the tapering profile can be observed with the increase in $\mathrm{Al}$ content from $0 \%$ to $100 \%$. The differences in etch rates are consistent with the higher binding energy of AlN, with bond energies of $11.52 \mathrm{eV} /$ atom for Al-N compared to 8.92 for Ga-N eV/atom $[18,19,20]$. Despite the higher volatility of $\mathrm{AlCl}_{\mathrm{x}}$ relative to $\mathrm{GaCl}_{\mathrm{x}}$, the comparison between $\mathrm{AlN}$ and GaN dry etching suggests that $\mathrm{AlCl}_{\mathrm{x}}$ is not easily formed due to the strong binding energy of AlN.

\subsection{Impact of etching conditions: time Etching} conditions with a good compromise between a high selectivity and a reasonable etch rate have been used to achieve higher-aspect-ratio AlN nanorods. Figure 5 illustrates the impact of etching time on the AlN nanorod morphology and etching selectivity. The values reported in Table 1 indicate that the AIN etch rate remains more or less the same whereas the selectivity drastically decreases by a factor of two between 14 and $30 \mathrm{~min}$. After $30 \mathrm{~min}$ etching, AlN nanorods with an aspect ratio greater than 6 can be achieved. However, such deep etching leads to the formation of increased SEM contrast along the sidewalls of the nanorod, interpreted as etching damage/roughness, and a tapering profile on the top part of the nanorod.

The reduced selectivity and increased tapering can be explained by the shrinkage of the metal mask. Indeed, the surface profile of the Ni dot implies a lower Ni thickness on its edges which will etch through quicker compared with the middle. Once this occurs, the shrinkage of the Ni dot will be progressively transferred into the AlN nanorod, manifested as a tapering of the sidewall. Therefore mask shrinkage will practically limit the aspect ratio of vertical-sided nanorods.

In addition to these observations, wrinkles can be observed at the bottom part of the 30 min etched nanorod (Figure 5.c). It is suspected that these defects are due to the initial AIN template quality. Indeed, the first growth steps of an AlN/sapphire template consist of the use of buffer layers having a 3D morphology and higher defect density [21]. The deeper the nanorod etching, the higher the probability will be for the nanorods to penetrate the AIN buffer layer. Therefore, in order to minimize the impact of the buffer layer on the quality of the bottom part of the nanorord, an intermediate etching of $21 \mathrm{~min}$ was chosen to create nanorod scaffolds having reasonable sidewall surface quality for MOVPE regrowth. The inset in Figure 5.b shows 
the AlN nanorod, after $\mathrm{Au} / \mathrm{Ni}$ removal and buffered oxide etch (BOE) 5:1 cleaning, subsequently used for MOVPE regrowth.
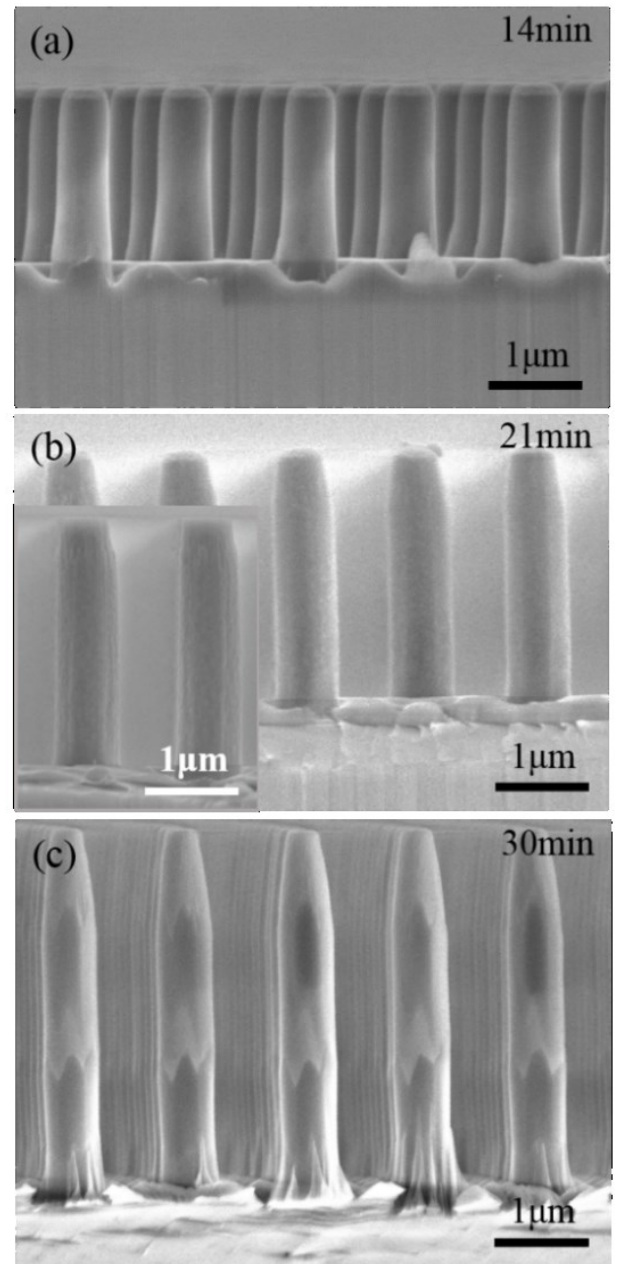

Figure 5 Cross-section SEM images of AIN nanorod arrays etched for various times. The inset in Figure (b) shows the AIN nanorods after Ni removal and BOE 5:1 cleaning.

3.4 Impact of regrowth conditions: continuous growth Figure 6 shows the effect of the temperature for a fixed pressure of $50 \mathrm{mbar}$ and a V/III ratio of 74.4. Independently of the growth temperature, the AlN regrowth that occurs on the top part of the nanorod leads to the formation of a truncated pyramid composed of six (10-11) semi-polar planes and a top $c$-plane. Along the nanorod sidewall, large striations are formed along the whole $m$ plane. In addition, almost no growth occurs along the $<11$ $20>a$-directions wich prevents any merging between neighbouring $m$-plane striated facets, and thus a complete facet recovery. The decrease of growth temperature from $1125{ }^{\circ} \mathrm{C}$ (Figure 6.a) to $1025{ }^{\circ} \mathrm{C}$ (Figure 6.b) mainly increases the $c$-plane and $m$-plane growth rate, but does not improve the facet recovery.
Extremly high growth temperatures are predicted to improve the surface morphology of $m$-plane AlN films [22], however such high temperatures were not accessible within the growth reactor employed. Thus, other parameters need to be investigated to improve the $m$-plane surface morphology and decrease the $m$-plane growth rate relative to the $a$-plane growth rate.
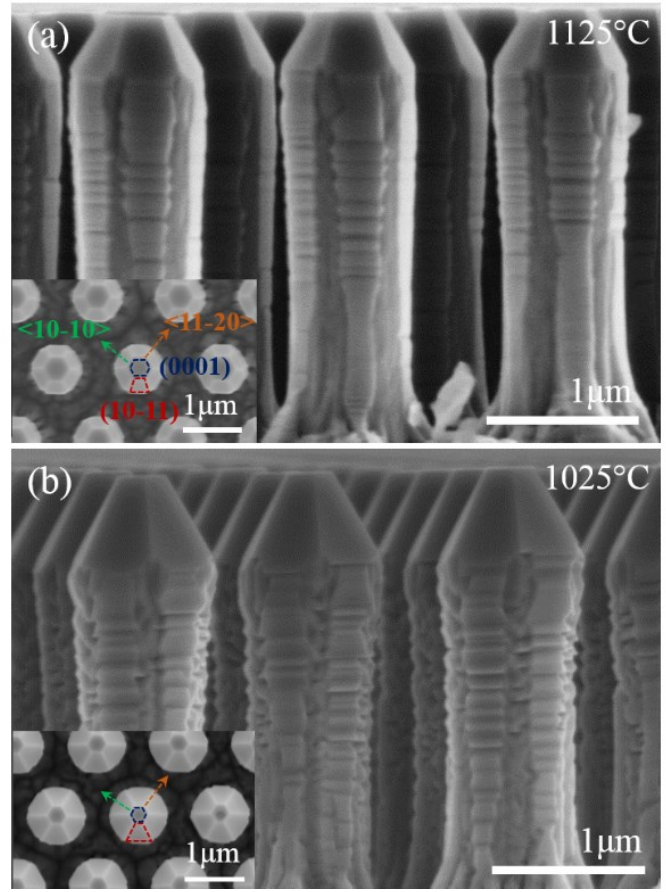

Figure 6 Cross-section SEM images of AIN nanorod after $90 \mathrm{~min}$ AlN regrowth at (a) $1125{ }^{\circ} \mathrm{C}$ and (b) $1025{ }^{\circ} \mathrm{C}$ with the following conditions fixed: $\mathrm{P}=50 \mathrm{mbar}, \mathrm{V} / \mathrm{III}=74.4(20 \mathrm{sccm} \mathrm{NH} 3$ and 20 sccm TMAl). The various planes/growth directions are indicated in the inset in Figure (a).

Figure 7 displays the nanorod morphology as a function of the pressure for a fixed temperature of $1125^{\circ} \mathrm{C}$ and a $\mathrm{V} / \mathrm{III}$ ratio of 3819. Firstly, the increase of V/III ratio from 74.4 (Figure 6.a) to 3819 (Figure 7.a) leads to smoother $m$ plane sidewalls and reduced $m$-plane growth in the upper part of the nanorod. However, there is no significant improvement of the growth along the $\langle 11-20>a$-directions. Secondly, the decrease of the pressure from $50 \mathrm{mbar}$ (Figure 7.a) to 20 mbar (Figure 7.b) further improves the $m$-plane surface quality, but most importantly, decreases the $m$-plane growth rate relatively to the $a$-plane growth rate, leaving a small gap between neighbouring $m$-plane facets. Additionally, at 20 mbar, the $c$-plane growth rate is increased which almost leads to a full pyramid with six (1011) semi-polar planes. It is also observed that inclined planes are formed in the upper part of the nanorod sidewalls, which could be associated with (n0-n-1) semipolar crystal planes, where $n \geq 1[9]$.

Thus, a lower pressure enhances $\mathrm{Al}$-adatom diffusion which almost leads to the merging of the neighbouring $m$ - 
plane facets. However, the same higher Al-adatom diffusion increases the incorporation of species at the junction between the semi-polar planes and $m$-planes. The resulting increase in diameter in the upper part of the nanorod then gradually prevents the growth species in the gas phase from diffusing towards the lower part of the nanorod and thus hinders complete facet recovery. Therefore, in order to reduce the $m$-plane growth in the upper part of the nanorod, higher V/III ratio values have been explored.
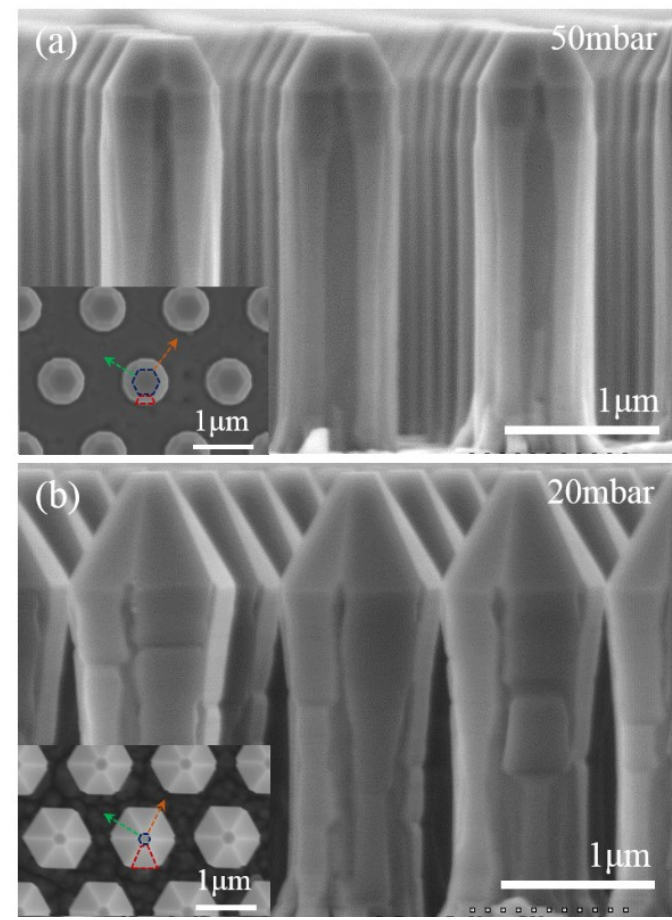

Figure 7 Cross-section SEM images of AlN nanorods after $90 \mathrm{~min}$ AlN regrowth at (a) 50 mbar and (b) 20 mbar with the following conditions fixed : $\mathrm{T}=1125^{\circ} \mathrm{C}, \mathrm{V} / \mathrm{III}=3819(1000 \mathrm{sccm} \mathrm{NH} 3$ and $20 \mathrm{sccm}$ TMAl).

Figure 8 illustrates the effect of increasing the V/III ratio on the AlN facet recovery for a fixed temperature of $1125^{\circ} \mathrm{C}$ and a pressure of 20 mbar. As the V/III ratio increases from 3819 to 15277 and 30554 , both the $c$-plane growth rate and the $m$-plane growth rate relatively to the $a$-plane growth rate decreases. At a V/III ratio of 30554 (Figure 8.b), the junction between neighbouring $m$-plane facets is completely formed in the whole upper part of the nanorod while a small gap is observed in the lower part. The drawback of such low $m$-plane growth rate is the high sensitivity to surface roughness. As observed in Figure 8.b, a large regrowth step can be observed in the upper part of each $m$-plane facets. This is due to the initial roughness observed on the top of the AlN etched nanorod (inset in Figure 5.b).
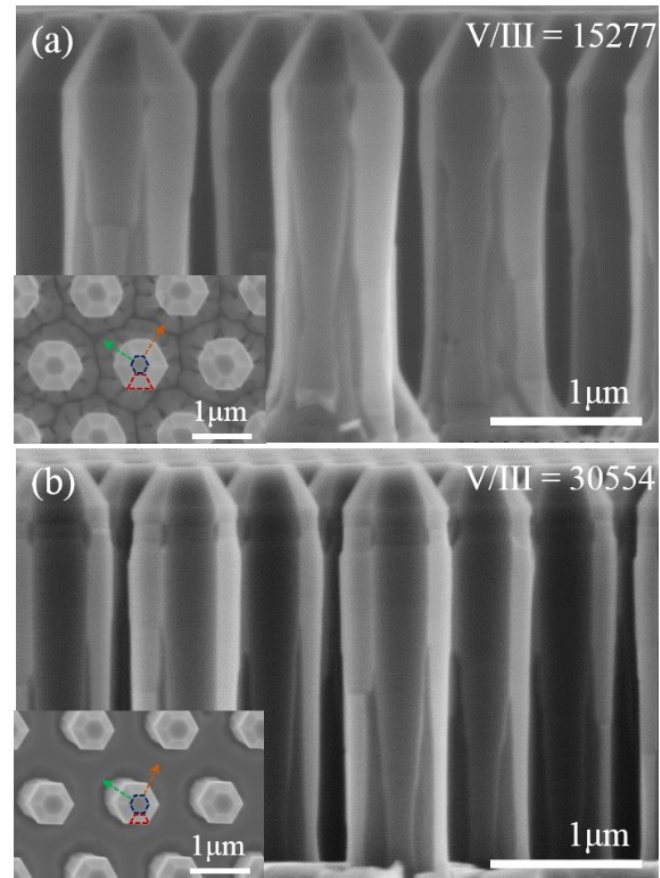

Figure 8 Cross-section SEM images of AlN nanorods after 90 min AlN regrowth with a V/III ratio of (a) 15277 and (b) 30554 with the following conditions fixed : $\mathrm{T}=1125^{\circ} \mathrm{C}, \mathrm{P}=20 \mathrm{mbar}$.

3.5 Impact of regrowth conditions: pulsed growth Various pulsed growth modes, as detailed in Figure 2 and Table 2, have been explored to further enhance the AlN facet recovery. All pulsed growths were performed under the same conditions as those presented in Figure 8.b. The $\mathrm{NH}_{3}$ pulsed growth mode (Figure 9.a) leads to an increase in the $m$-plane surface roughness along with a low growth rate along the $\langle 11-20\rangle$ direction, consistent with the results for a lower effective V/III ratio. The modified MEE (Figure 9.b) only improves the surface quality of the $m$ plane facets. The TMAl pulsed growth further improves the surface quality and leads to the formation of a junction between neighbouring $m$-plane facets in the upper part of the nanorod. However, similarly to Figure 8.b, regrowth steps can be observed on the top part of each $m$-plane facet.

Therefore, within the employed growth conditions, the use of a $\mathrm{NH}_{3}$ pulsed growth mode decreases the Al-adatom diffusion along the sidewalls along with the $m$-plane growth rate relative to the $a$-plane growth rate. Similar conclusions can be deduced for the modified MEE approach. The TMAl pulsed growth gives the best results. This could be expected since facet recovery is improved for high V/III ratio. The use of TMAl pulsed growth effectively increases the V/III ratio by saturating the surface with $\mathrm{N}$-adatoms. 

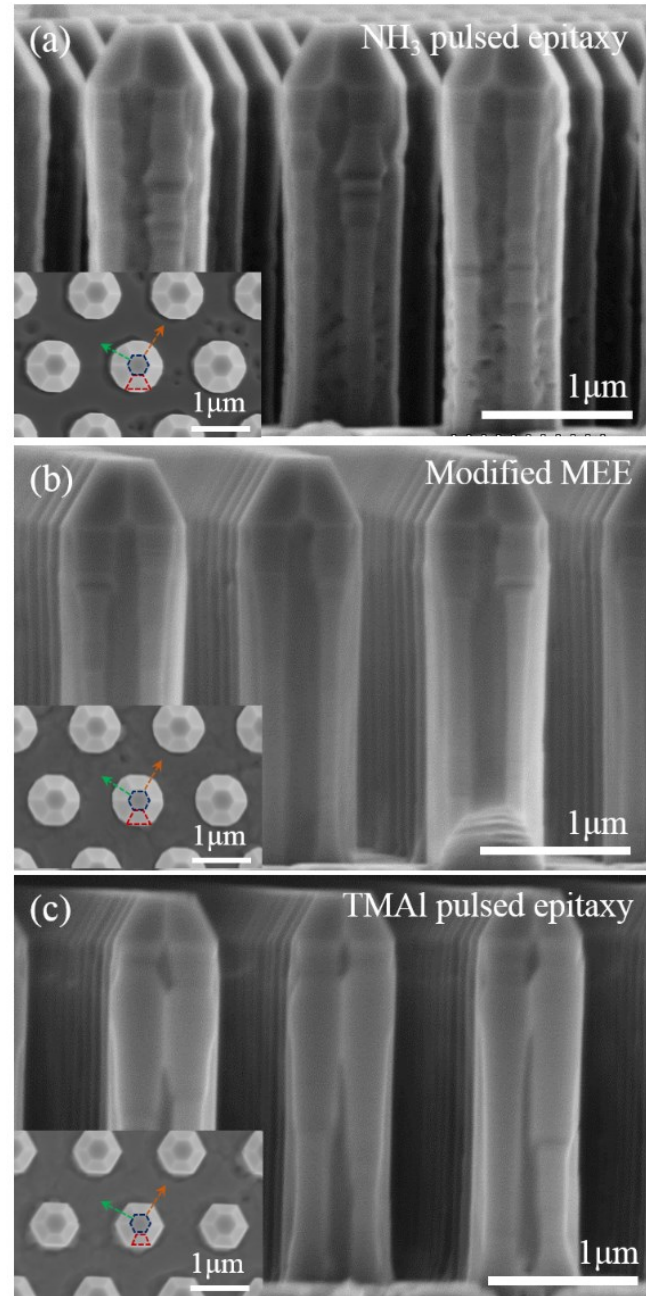

Figure 9 Cross-section SEM images of AIN nanorods after 90 min AlN regrowth for (a) $\mathrm{NH}_{3}$ pulsed epitaxy, (b) modified migration enhanced epitaxy (MEE) and (c) TMAl pulsed epitaxy with the following conditions fixed : $\mathrm{T}=1125^{\circ} \mathrm{C}, \mathrm{P}=20 \mathrm{mbar}, \mathrm{V} / \mathrm{III}=$ 30554 (4000 sccm NH 3 and $10 \mathrm{sccm} \mathrm{TMAl).}$

3.6 Discussion The various growth conditions and growth modes employed in sections 3.4 and 3.5 show that the growth parameter window to achieve AIN facet recovery is narrow. The use of a high V/III ratio or TMAl pulsed growth is not conventional for $m$-plane planar growth [22] or $c$-plane planar growth performed on various substrates/templates $[7,23,24,25]$. But within a nanorod geometry, up to four different facets can co-exist [26], so that competition between various growth directions occurs. In this study, we have demonstrated that the change in V/III ratio has a major impact on the AlN regrowth along $<0001>$, $<10-10>$ and $<11-20>$. In particular, AIN facet recovery along the sidewall is highly improved for high V/III ratio, where the $c$-plane and $m$-plane growth rate is lowered relative to the $a$-plane growth rate.

Further improvement in the AlN facet recovery should be achieved by optimizing the fabrication process. First, $\mathrm{KOH}-$ based wet etching will improve the sidewall surface morphology of the nanorod after dry etching [27]. This will prevent the formation of regrowth steps as observed in Figure 8.b and Figure 9.c. Second, the introduction of a passivation layer at the bottom of etched nanorods can avoid the incorporation of adatoms on the $c$-plane surface [28]. This could result in a higher incorporation of adatoms along the sidewalls, especially in the lower part of the nanorod where the junction between neighbouring $m$-planes is not complete. Finally, a major improvement should be expected by tuning the aspect ratio of the AlN etched nanorods. An increase of the pitch should result in more adatom incorporation on the nanorod sidewalls; a decrease of the nanorod height could mitigate the lack of growth along the $\langle 11-20\rangle$ direction in the lower part of the nanorod; and most importantly, a decrease of the initial diameter is predicted to induce the formation of a junction between two neighbouring $m$-plane more quickly.
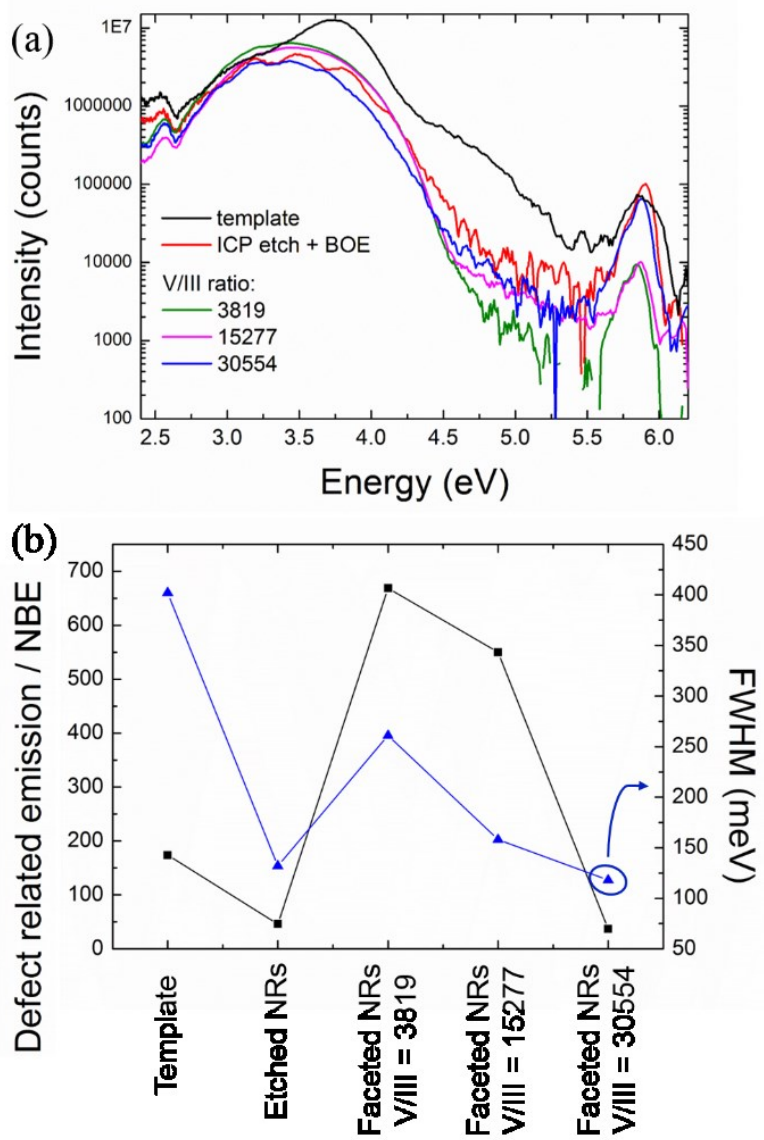

Figure 10 (a) RT cathodoluminescence spectra in log scale acquired on AlN template (black spectrum), AlN etched nanorod (red spectrum) and AlN faceted nanorod with a V/III ratio of 3819 (green spectrum), 15277 (purple spectrum) and 30554 (blue spectrum). (b) Ratio between the defect-related emission and AIN NBE (in black and on the left axis) and FWHM of the NBE (in blue and on the right axis) for the AlN template, AlN etched nanorod and AlN faceted nanorod with a V/III ratio of, 15277 and 30554. 
Author, Author, and Author: Short title

3.7 AIN nanorod optical properties Cathodoluminscence spectra were acquired at room temperature on the AlN template, the AlN etched nanorods and the AIN faceted nanorods grown at various V/III ratios. For the nanorod samples, the spectra were recorded from positions half way up the nanorod sidewalls. Figure 10.a shows the presence of the AlN near band-edge emission (NBE) centred around 5.9 $\mathrm{eV}[29,30]$ along with a broad emission band between 2.6 $\mathrm{eV}$ and $4.3 \mathrm{eV}$ for all spectra. The various contributions observed in the broad emission band could be related to $\mathrm{O}$ complexes and/or Si-complexes and other native defects, such as vacancies $[30,31]$. A significant reduction of the deep broad emission band in the etched and faceted AlN nanorods was observed, in particular for the contribution at $3.75 \mathrm{eV}$. The defect related emission / NBE ratio (Figure 10.b) was highly improved after ICP etching of the nanorod and after subsequent AlN faceting at high V/III ratio. Lower V/III was found to increase the defect related emission / NBE ratio. A similar trend was observed for the full width at half maximum (FWHM) of the AIN NBE, with the smallest values associated with the etched nanorods and the AlN faceted nanorods at high V/III ratio.

The improvement in the emission spectrum after ICP etching was surprising, but could be attributed to an increased extraction efficiency that would affect the NBE more than the lower energy emission due to the band absorption tail and/or the reduction of strain after etching affecting the nature of the valence band edge. The improved

${ }^{1}$ M. Kneissl and J. Rass, Springer Series in Materials Science, Vol. 227 (2016)

${ }^{2}$ Y. Narukawa, M. Ichikawa, D. Sanga, M. Sano, T. Mukai, J. Phys D Appl. Phys. 43, 354002 (2010)

${ }^{3}$ M. Kneissl, T. Kolbe, C. Chua, V. Kueller, N. Lobo, J. Stellmach, A. Knauer, H. Rodriguez, S. Einfeldt, Z. Yang, N.M. Johnson, M. Weyers, Semicond. Sci. Technol. 26, 014036 (2011)

${ }^{4}$ W. Bergbauer, M. Strassburg, Ch. Kölper, N. Linder, C. Roder, J. Lähnemann, A. Trampert, S. Fündling, S. F. Li, H. H. Wehmann, and A. Waag, Nanotechnology 21, 305201 (2010).

${ }^{5}$ K. Choi, M. Arita, and Y. Arakawa, J. Cryst. Growth 357, 58 (2012).

${ }^{6}$ P. M. Coulon, B. Alloing, V. Brändli, D. Lefebvre, S. Chenot, and J. Zúñiga-Pérez, Phys. Status Solidi B 252, 1096 (2015).

${ }^{7}$ R. G. Banal, M. Funato and Y. Kawakami, Phys. Status Solidi C 6, 599-602 (2009)

${ }^{8}$ E.D. Le Boulbar, P.R. Edwards, S.H. Vajargah, I. Griffiths, I. Gîrgel, P.-M. Coulon, D. Cherns, R.W. Martin, C.J. Humphreys, C.R. Bowen, D.W.E. Allsopp, P.A. Shields, Cryst. Growth Des. 16, 1907-1916 (2016)

${ }^{9}$ Y. Tian, J. Yan, Y. Zhang, Y. Zhang, X. Chen, Y. Guo, J. Wang and J. Li, Nanoscale 8, 11012-11018 (2016)

${ }^{10}$ Q. Li, J. J. Figiel and G. T. Wang, Appl. Phys. Lett. 94, 231105 (2009).

${ }^{11}$ A. Waag, X. Wang, S. Fundling, J. Ledig, M. Erenburg, R. Neumann, M. Al Suleiman, S. Merzsch, J. Wei, S. Li, H. H. Wehmann, W. Bergbauer, M. Straßburg, A. Trampert, U. Jahn, and H. Riechert, Phys. Status Solidi C 8, 2296 (2011). spectrum with higher V/III ratio could be attributed to improved material quality through reduced defect incorporation. All these observations illustrate first an improvement of the optical quality after dry etching, and second that a high V/III ratio faceting regrowth is appropriate to achieve AlN faceted nanorod with good optical quality.

4 Conclusion In summary, the route towards the fabrication of regular arrays of straight AlN-faceted nanorods has been investigated and discussed for a hybrid top-down/bottom-up approach. The use of a relatively high pressure during ICP dry etching is found to improve the nanorod sidewall profile and selectivity. The subsequent AlN facet recovery is promoted at low pressure and extremly high V/III ratio. The resulting smooth and straight non-polar facets exhibit higher optical properties compared to the initial template. Finally, we proposed that by optimizing the aspect ratio of the etched nanorods the AlN facet recovery will be further improved. Such fabrication of straight, faceted, AIN nanorod arrays via a hybrid topdown/bottom-up is the first step towards high-efficiency deep-UV-emitting core-shell LEDs.

Acknowledgements The authors would like to acknowledge financial support from the EPSRC, UK via Grant Nos. EP/M015181/1 \& EP/M022862/1, "Manufacturing nanoGaN". The data associated with this research are available at https://doi.org/10.15125/BATH-00415 or from the corresponding author.

\section{References}

${ }^{12}$ M. Djavid and Z. Mi, Appl. Phys. Lett. 108, 051102 (2016)

${ }^{13} \mathrm{http}: / /$ www.nanowin.com.cn/en/show_cpzs.asp?Id=9

${ }^{14}$ P. Shah Amit, R. Laskar Masihhur, A. Azizur Rahman, R. Gokhale Maheshwar, and Arnab Bhattacharya, J. Vac. Sci. Technol. A 31, 061305 (2013)

${ }^{15}$ K. Zhu, V. Kuryatkov, B. Borisov, J. Yun, G. Kipshidze, S. A. Nikishin, and H. Temkin, D. Aurongzeb and M. Holtz, J. Appl. Phys. 95, 4635 (2004)

${ }^{16}$ E.D. Le Boulbar, C.J. Lewins, D.W.E. Allsopp, C.R. Bowen and P.A. Shields, Microelectronic Engineering 153, 132-136 (2016)

${ }^{17}$ P.R. Edwards , L.K. Jagadamma , J. Bruckbauer, C. Liu , P. Shields , D. Allsopp , T. Wang and R.W. Martin, Microsc. Microanal. 18, 1212-1219 (2012)

${ }^{18}$ R. J. Shul, R. D. Briggs, S. J. Pearton, C. B. Vartuli, C. R. Abernathy, J. W. Lee, C. Constantine, and C. Barratt, Mater. Res. Soc. Symp. Proc. 449, 969 (1996).

${ }^{19}$ A. Costales, M. A. Blanco, A. M. Pendas, A. K. Kandalam, and R. Pandey, J. Am. Chem. Soc. 124, 4116 (2002).

${ }^{20}$ H. Morkoc, Handbook of Nitride Semiconductors and Devices, Wiley-VCH, (2008)

${ }^{21}$ N. Okadaa, N. Katoa, S. Satoa, T. Sumiia, T. Nagaia, N. Fujimotoa, M. Imuraa, K. Balakrishnana, M. Iwayaa, S. Kamiyamaa, H. Amanoa, I. Akasakia, H. Maruyamab, T. Takagib, T. Norob, A. Bandohc, Journal of Crystal Growth 298, 349-353 (2007)

${ }^{22}$ I. Bryan, Z. Bryan, M. Bobea, L. Hussey, R. Kirste, R. Collazo, and Z. Sitar. J. Appl. Phys. 116, 133517 (2014) 
${ }^{23}$ Z. Chen, , S. Newman, D. Brown, R. Chung, S. Keller, U. K. Mishra, S. P. Denbaars, and S. Nakamura, Appl. Phys. Lett. 93, 191906 (2008)

${ }^{24}$ Y. Feng, H. Wei, S. Yang, Z. Chen, L. Wang, S. Kong, G. Zhao and X. Liu, Sci. Rep. 4, 6416 (2014).

${ }^{25}$ I. Bryan, Z. Bryan, S. Mita, A. Rice, J. Tweedie, R. Collazo, and Z. Sitar, Journal of Crystal Growth 438, 81-89 (2016).

${ }^{26}$ I. Gîrgel , P. R. Edwards , E. Le Boulbar, P.-M. Coulon, S.-L. Sahonta, D. W. E. Allsopp, R. W. Martin, C. J. Humphreys, and P. A. Shields , J. Nanophotonics 10, 16010 (2016). $\begin{array}{ll}11 & 27 \\ 12 & \text { Qester, Li, J. B. Wright, W. W. Chow, T. S. Luk, I. Brener, L. F. }\end{array}$

$\begin{array}{ll}11 & 27 \text { Q. Li, J. B. Wright, W. W. Chow, T. S. Luk, I. Brener, L. F. } \\ 12 & \text { Lester, and G. T. Wang, Opt. Express 20, } 17873 \text { (2012). }\end{array}$
${ }^{28}$ E. D. Le Boulbar, I. Gîrgel, C. J. Lewins, P. R. Edwards, R. W. Martin, A. Šatka, D. W. E. Allsopp, and P. A. Shields, Journal of Applied Physics 114, 094302 (2013)

${ }^{29}$ E. Silveira, J.A. Freitas, S.B. Schujman, L.J. Schowalter, Journal of Crystal Growth 310, 4007-4010 (2008)

${ }^{30}$ Z. Bryan, I. Bryan, M. Bobea, L. Hussey, R. Kirste, Z. Sitar, and Ramon Collazo, J. Appl. Phys. 115, 133503 (2014)

${ }^{31}$ A. Dadgar, A. Krost, J. Christen, B. Bastek, F. Bertram, A. Krtschil, T. Hempel, J. Blasing, U. Haboeck, A. Hoffmann, Journal of Crystal Growth 297, 306-310 (2006) 